\title{
La eficacia de los sistemas intrauterinos con levonorgestrel no sería inferior a la de los dispositivos intrauterinos de cobre para la anticoncepción de emergencia
}

The efficacy of levonorgestrel intrauterine systems would not be inferior to that of copper intrauterine devices for emergency contraception

\section{Comentado de:}

Turok DK, et al. N Engl J Med. 2021;384:335-44. PMID: $33503342^{1}$

\section{Objetivo}

Evaluar el riesgo de embarazo a un mes con un dispositivo intrauterino (DIU) de 52 mg de levonorgestrel en comparación con un DIU de cobre para la anticoncepción de emergencia.

\section{Diseño, lugar y pacientes}

Ensayo aleatorizado multicéntrico de no inferioridad, llevado a cabo en seis clínicas en Utah, EE.UU., entre 2016 y 2019.

Se incluyeron mujeres que buscaron anticoncepción de emergencia después de al menos un episodio de relaciones sexuales sin protección dentro de los cinco días anteriores a la consulta y que aceptaron la colocación de un DIU.

Se asignó aleatoriamente a las participantes en una proporción de 1:1 para recibir un DIU de $52 \mathrm{mg}$ de levonorgestrel (sitema intrauterino, SIU) o un DIU de cobre T380A (DIU-Cu).

Se eligió el diseño de no inferioridad debido a la preferencia por el SIU mostrado por las participantes en ensayos anteriores; establecer su no inferioridad frente al DIU-Cu para la anticoncepción de emergencia podría aumentar las opciones de las pacientes ante esa situación.

\section{Medición de resultados principales}

El resultado primario fue una prueba de embarazo en orina positiva a un mes de la inserción del dispositivo. Cuando este resultado no estaba disponible se utilizaron datos de encuestas y registros médicos para determinar un embarazo.

\section{Resultados Principales}

Un total de 711 mujeres participaron de la aleatorización. Entre las 355 participantes asignadas al azar para recibir SIU y 356 asignadas para recibir DIU-Cu, 317 y 321 , respectivamente, recibieron las intervenciones y proporcionaron datos de resultados de un mes de seguimiento; 290 en el grupo SIU y 300 en el grupo DIU-Cu realizaron una prueba de embarazo en orina en ese plazo.

En los análisis modificados por intención de tratar y por protocolo, las tasas de embarazo fueron de 1 en 317 (0,3\%; intervalo de confianza [IC] del $95 \%, 0,01$ a 1,7$)$ en el grupo de SIU y 0 en 321 (0\%; IC $95 \%, 0$ a 1,1) en el grupo de DIU-Cu. La diferencia absoluta entre grupos en ambos análisis fue de 0,3 puntos porcentuales (IC $95 \%,-0,9$ a 1,8), coherente con la no inferioridad del SIU frente al DIU-Cu (el margen de no inferioridad preespecificado fue de 2,5 puntos porcentuales) (ver Tabla 1).

El $5,2 \%$ de los participantes en el grupo de SIU y $4,9 \%$ en el grupo de DIU-Cu tuvieron un evento adverso que provocó la búsqueda de atención médica durante el primer mes después de la colocación.

Tabla 1. Incidencia de embarazo en los grupos evaluados al mes de seguimiento según tipo de análisis utilizado

\begin{tabular}{|l|c|c|c|}
\hline Enfoque de Análisis & SIU de $52 \mathrm{mg}$ de levonorgestrel, n/N & DIU-Cu, n/N & $\begin{array}{c}\text { Diferencia de incidencia } \\
\text { SIU-DIU-Cu (IC 95\%) }\end{array}$ \\
\hline Intención de tratar & $1 / 317$ & $0 / 321$ & $0,3(-0,9$ a 1,8$)$ \\
\hline Por protocolo & $1 / 305$ & $0 / 311$ & $0,3(-0,9$ a 1,8$)$ \\
\hline Análisis de sensibilidad & $1 / 290$ & $0 / 300$ & $0,3(-0,9$ a 1,9$)$ \\
\hline
\end{tabular}

\section{Conclusiones}

EI SIU con levonorgestrel no fue inferior al DIU-Cu para la anticoncepción de emergencia.

Fuente de financiamiento/Conflicto de interés de los autores: Estudio financiado por subsidios y premios provenientes del Instituto Nacional de
Salud del Niño y el Desarrollo Humano Eunice Kennedy Shriver, la Universidad de Utah, y los Institutos Nacionales de Salud de los EE.UU. Los autores declararon no tener conflictos de interés, excepto el autor principal quien se desempeña como director de servicios quirúrgicos de Planned Parenthood Association of Utah, institución en la que se reclutaron las participantes en el estudio. 


\section{Comentario}

Este estudio evalúa la no inferioridad del SIU con levonorgestrel respecto al DIU-Cu para la anticoncepción de emergencia Los autores parten de evidencia previa que indica que, al menos en los EE.UU., cada vez más usuarias de dispositivos intrauterinos eligen el SIU por sobre los DIU-Cu para la anticoncepción a largo plazo. Por otro lado, en un estudio previo del mismo autor ${ }^{2}$ las usuarias mostraron preferencia por el SIU sobre el DIU-Cu en situación de anticoncepción de emergencia, y no se informaron embarazos entre las 121 personas que tomaron anticonceptivos orales de emergencia de levonorgestrel y también se colocaron rápidamente un SIU.

La principal limitación de este ensayo es que de las 10.317 personas que buscaron anticoncepción de emergencia que fueron evaluadas para su elegibilidad, solo $7 \%$ ingresaron al estudio ( $80 \%$ se negó a participar y $13 \%$ no cumplía con los criterios de inclusión), lo que podría dar lugar a limitaciones en la generalizabilidad de los resultados, por tratarse de una población en estudio altamente seleccionada.

Por otro lado, los médicos del estudio estaban al tanto del tipo de DIU que recibieron las participantes, y algunas de ellas atendidas en la clínica para la visita de seguimiento luego del primer mes, pudieron haber sido informadas sobre la intervención antes de completar la encuesta de seguimiento. Aun así, el embarazo se considera un desenlace lo suficientemente objetivo para que las fallas en el cegamiento de las participantes tuviera un efecto sobre el resultado.

Es probable que se necesite mayor evidencia para incorporar modificaciones en la práctica cotidiana. Por ej., la Unidad de Efectividad Clínica de la Facultad de Salud Sexual y Reproductiva, organización que establece los estándares para los médicos de planificación familiar y salud sexual en el Reino Unido, no recomienda ningún cambio en la práctica actual aún luego de la publicación de este trabajo ${ }^{3}$. Sin embargo, los hallazgos presentados sugieren que el SIU podría ser un método eficaz de anticoncepción de emergencia, por lo que se recomienda realizar más investigaciones.

\section{Conclusiones de la comentadora}

En Argentina queda pendiente la incorporación del DIU-Cu como anticonceptivo de emergencia, ya que a pesar de su probada efectividad, el método más utilizado es la anticoncepción oral con levonorgestrel ${ }^{4,5}$. Por otro lado, en este ámbito, el elevado costo podría ser una de las principales barreras para la utilización del SIU con este fin.

Natalia Mandel [ Servicio de Medicina Familiar y Comunitaria, Hospital Italiano de Buenos Aires. natalia.mandel@hospitalitaliano.org.ar ]

Mandel N La eficacia de los sistemas intrauterinos con levonorgestrel no sería inferior a la de los dispositivos intrauterinos de cobre para la anticoncepción de emergencia. Evid Actual Pract Ambul. 2021;24(3):e002132. Available from: https://dx.doi.org/10.51987/EVIDENCIA.V24I4.6956. Comentado de: Turok DK, et al. Levonorgestrel vs. Copper Intrauterine Devices for Emergency Contraception. N Engl J Med. 2021 Jan 28;384(4):335-344. doi: 10.1056/NEJMoa2022141. PMID: 33503342

\section{Referencias}

1. Turok DK, Gero A, Simmons RG, et al. Levonorgestrel vs. Copper Intrauterine Devices for Emergency Contraception. N Engl J Med. 2021;384(4):335344. Available from: 10.1056/NEJMoa2022141.

2. Turok DK, Sanders JN, Thompson IS, et al. Preference for and efficacy of oral levonorgestrel for emergency contraception with concomitant placement of a levonorgestrel IUD: a prospective cohort study. Contraception. 2016;93(6):526-532. Available from: 10.1016/j.contraception.2016.01.009;https: //dx.doi.org/10.1016/j.contraception.2016.01.009.

3. FSRH CEU Statement: Response to Recent Publication Turok et al. (2021) ; 2021. Available from: https://www.fsrh.org/standards-and-guidance/ documents/fsrh-ceu-statement-response-to-recent-publication-turok-et-al/.

4. Anticoncepcion de urgencia; 2018. Available from: https://www.who.int/es/news-room/fact-sheets/detail/emergency-contraception\#: :text=y\% 20sumamente\%20eficaz.- ,Eficacia [Last access: 2021-06-23].

5. Cleland K, Zhu H, Goldstuck N, et al. The efficacy of intrauterine devices for emergency contraception: a systematic review of 35 years of experience. Human Reproduction. 2012;27(7):1994-2000. Available from: 10.1093/humrep/des140; https://dx.doi.org/10.1093/humrep/des140. 